\title{
Rôle des inspecteurs de l'enseignement secondaire en Communauté française de Belgique
}

Jean Ravez

\section{(2) OpenEdition}

Édition électronique

URL : http://journals.openedition.org/ries/3557

DOI : 10.4000/ries.3557

ISSN : 2261-4265

Éditeur

Centre international d'études pédagogiques

Édition imprimée

Date de publication : 1 décembre 1995

Pagination : 129-131

ISSN : 1254-4590

\section{Référence électronique}

Jean Ravez, «Rôle des inspecteurs de l'enseignement secondaire en Communauté française de Belgique », Revue internationale d'éducation de Sèvres [En ligne], 08| 1995, mis en ligne le 14 février 2014, consulté le 01 mai 2019. URL : http://journals.openedition.org/ries/3557 ; DOI : 10.4000/ ries.3557

Ce document a été généré automatiquement le 1 mai 2019.

(c) Tous droits réservés 


\title{
Rôle des inspecteurs de l'enseignement secondaire en Communauté française de Belgique
}

\author{
Jean Ravez
}

1 Notre enseignement est organisé en « réseaux » et les missions assignées aux inspecteurs diffèrent selon l'appartenance des écoles où ils sont appelés à intervenir.

2 Les établissements relèvent de l'un des trois réseaux mentionnés ci-dessous :

- le réseau officiel de la Communauté (naguère «enseignement de l'État») organisé sous la responsabilité directe du ministre de l'éducation,

- le réseau subventionné officiel dont les écoles sont organisées par d'autres pouvoirs publics (les provinces et les communes pour l'essentiel) qui reçoivent des subsides si elles se conforment aux dispositions légales et réglementaires en vigueur (par exemple en matière de programmes, d'horaires, d'adéquation des locaux, de l'équipement et du matériel didactique),

- le réseau subventionné libre, quasi exclusivement catholique, et dont les établissements sont subsidiés dans les mêmes conditions que les écoles relevant du réseau subventionné officiel.

Dans les réseaux subventionnés (officiel et libre), la tâche principale des inspecteurs est d'examiner si les écoles dispensent un enseignement conforme aux conditions requises pour recevoir des subsides. Il s'agit notamment de déterminer si les études se situent à un niveau suffisant et si les programmes approuvés par le ministre sont suivis.

4 Pour exercer cette mission de contrôle, les inspecteurs peuvent assister aux cours, interroger les élèves, examiner leurs travaux, prendre connaissance des documents dont la tenue est requise, visiter les locaux...

5 Ils doivent cependant s'abstenir de formuler des directives en matière de méthodes pédagogiques : elles sont laissées à l'initiative des pouvoirs organisateurs.

6 En revanche, dans le réseau géré par le ministre, les inspecteurs interviennent dans le choix des méthodes. Outre les avis qu'ils formulent sur l'activité individuelle des professeurs, ils ont pour mission de les conseiller, d'assurer l'animation des équipes 
pédagogiques, d'organiser ou de superviser la formation en cours de service des enseignants et, dans le domaine technique, d'établir une collaboration permanente avec les milieux socio-professionnels.

7 Dans ce réseau, le corps inspectoral exerce à la fois des tâches inhérentes au contrôle et des tâches qui ressortissent à la guidance. Ces deux activités sont complémentaires. D'une part, on imagine mal qu'un éventuel constat de carence ne donne pas lieu à des conseils concrets visant à améliorer la qualité de l'enseignement. D'autre part, les suggestions formulées par l'inspecteur risqueraient de rester lettre morte pour certains, en l'absence de perspective d'une vérification ultérieure.

Il y a lieu de signaler que la mission de contrôle individuel des enseignants, qui était jadis considérée comme primordiale, tend à perdre de son importance au profit des activités d'animation et de guidance.

9 Les inspecteurs sont aussi responsables de l'élaboration des programmes soumis à l'approbation ministérielle ainsi que de la rédaction des instructions qui s'y rapportent. Pour ce faire, ils assurent la présidence de commissions composées de professeurs et, le cas échéant, d'experts dont ils sollicitent la collaboration. Le rôle de l'inspection est prépondérant lors de la mise en œuvre des réformes pédagogiques (information des professeurs, réunion de groupes de travail, journées de formation).

De manière très générale, les inspecteurs doivent s'acquitter de toute mission, qui leur est confiée, par le ministre ou par les instances administratives. Il leur est, par exemple, demandé de mener des procédures d'enquête lorsqu'un enseignant est mis en cause dans l'exercice de sa profession, quand des dysfonctionnements apparaissent dans l'organisation d'une école, quand des parents introduisent une plainte...

11 Le corps inspectoral est ainsi amené à exercer des activités diverses et multiples, mais qui sont en fait complémentaires puisqu'elles relèvent pour l'essentiel du domaine pédagogique.

Son rôle est devenu d'autant plus important que les écoles disposent d'une marge d'autonomie de plus en plus grande et qu'il importe de garantir la cohérence du système éducatif général.

13 Ce rôle, les inspecteurs peuvent l'assumer efficacement dans la mesure où ils sont recrutés à la suite d'épreuves d'aptitude à la fonction et compte tenu de leur compétence pédagogique et scientifique, dans la mesure aussi où ils disposent d'une connaissance directe des problèmes rencontrés sur le terrain, dans la mesure enfin où ils constituent le trait d'union entre le pouvoir central et les communautés scolaires.

14 Une des difficultés rencontrées par l'inspection telle qu'elle est organisée en Communauté française de Belgique trouve son origine d'une part, dans le fait que les inspecteurs (comme la plupart des enseignants du secondaire!) se perçoivent d'abord comme des spécialistes d'une discipline déterminée. Cet état d'esprit ne va pas sans porter préjudice à la mise en œuvre des approches pluridisciplinaires dont nul ne songerait plus aujourd'hui à contester l'absolue nécessité.

15 D'autre part, il serait souhaitable que l'inspection procède de manière systématique à l'audit d'établissements. Toutefois, la simple juxtaposition des constats établis pour chaque discipline ne suffit pas pour évaluer correctement l'action éducative développée dans une école. À cet égard, nous regrettons de ne pas pouvoir disposer d'inspecteurs « de la vie scolaire", qui pourraient intervenir à bon escient en matière d'organisation 
générale des établissements, de contacts avec les parents, de relations entre la direction et le personnel...

De manière plus large, il faudrait que l'inspection ait des moyens suffisants pour prendre en charge le pilotage du système éducatif. C'est d'autant plus nécessaire que les inspecteurs sont aussi appelés à jouer le rôle de conseiller des ministres, rôle qu'ils ne peuvent exercer pleinement s'ils ne sont pas en mesure de procéder à des investigations présentant les garanties scientifiques requises.

17 À cet égard, il importe que le corps inspectoral jouisse d'une autonomie qui préserve l'objectivité des données et des propositions qu'il soumet à l'autorité politique.

18 Au moment où le système éducatif connaît de profondes mutations, il faut que le ministre dispose d'un corps de spécialistes qui puisse - en toute indépendance par rapport aux structures administratives - l'éclairer sur l'utilisation des crédits consacrés à la formation des élèves, l'informer concernant l'efficacité de l'enseignement et assurer le respect des objectifs généraux fixés par le législateur.

\section{RÉSUMÉS}

L'organisation de l'enseignement en trois réseaux détermine les missions assignées aux inspecteurs. Dans les deux réseaux subventionnés, elles se limitent au contrôle. Elles sont plus larges dans le réseau officiel géré par le ministre et concernent le contrôle, le conseil et l'élaboration des programmes. Les mutations du système éducatif rendraient nécessaires le recours à des approches pluridisciplinaires et la pratique d'audit d'établissement.

\section{INDEX}

Index géographique : Belgique, Belgique francophone, Communauté française de Belgique Mots-clés : inspection, système éducatif

\section{AUTEUR}

\section{JEAN RAVEZ}

Inspecteur général, ministère de l'éducation, Bruxelles, Belgique. 